\title{
A novel three-dimensional printed vacuum bell for pectus excavatum treatment: a preliminary study
}

Xicheng Deng ${ }^{1 *}$, Peng Huang ${ }^{1}$, Jinwen Luo ${ }^{1}$, Jinghua Wang ${ }^{1}$, Liwen $\mathrm{Yi}^{1}$, Guangxian Yang ${ }^{1}$, Siping $\mathrm{He}^{2}$, Xun Li ${ }^{3}$ and Shiting Xiang ${ }^{3}$

\begin{abstract}
Objective: Conservative treatment with a vacuum bell (VB) for pectus excavatum (PE) has now been gradually popularized as an alternative to surgery. We describe our initial experience with a novel three dimensional (3D) printed VB device.

Methods: Prospectively collected data of all patients who started using a 3D printed VB in 2018 at our institution were analyzed. Linear and logistic regressions were used to identify factors associated with effectiveness of device usage.

Results: In total, forty-two patients with a median age of 3.6 years were treated with the device. The median follow-up duration was 11.1 months and the mean initial Depth Ratio (DR) was 0.129. There were no permanent sequelae from side effects. Thirty patients with at least one follow-up body scan data showed varying improvement $(z=-4.569, p=0.0000)$. Linear regression suggested that longer usage improved outcomes $\left(R^{2}=0.235, p=0.014\right)$. By logistic regression there was a trend of younger ages and less initial DR for better improvement though neither was statistically significant $(p=0.086$, 0.078 , respectively).

Conclusion: Our initial experience has shown the 3D printed VB may be as effective as other conventional VBs and could be used as an alternative to surgical treatment for selected patients with PE. More experience and studies with this type of $\mathrm{VB}$ are needed to demonstrate its superiority with regard to the 3D printing design and optimal timing and indication for use.
\end{abstract}

Keywords: Pectus excavatum, Vacuum bell, 3D printed

\section{Background}

The vacuum bell $(\mathrm{VB})$ has established its role in the treatment of pectus excavatum (PE) in the last 20 years [1-6] as an alternative to surgery it has been extensively reported by Haecker, et al. [1-3]. And then its usefulness has been confirmed by other authors [4]. However, all their VBs are premade with fixed sizes and shape. In this study, we tried to incorporate the unique configuration of the chest wall

\footnotetext{
* Correspondence: justindxc@gmail.com

${ }^{1}$ Deaprtment of Cardiothoracic Surgery, Hunan Children's Hospital, No. 86 Ziyuan Road, Changsha 410007, Hunan, China

Full list of author information is available at the end of the article
}

deformity of each and every individual for the manufacture of a VB. We started to use a three dimensional (3D) printed VB bespoke for every patient since 2018. To the best of our knowledge, this is the first one of its kind incorporating 3D technique. Here we report our initial experience with this novel VB and short-term follow-up results.

\section{Methods}

The manufacture of a 3D printed VB

The 3D printed VB (Kenvoxel Co.,Ltd. Guangzhou, China) is manufactured involving the optical body surface scan steps and has been described in detail in our 
previous publication [7]. Once data has been obtained with body surface scan, the images are imported to Geomagic Studio (3D Systems ${ }^{\bullet}$, Rock Hill, SC, USA) to extract the funnel area, ready for design of the silicon part (skin contact part) (Fig. 1). The silicon part is manufactured and connected to a top part with an electronic pressure gauge and a check valve (for hand pump connection) mounted and ready for use. (Fig. 2).

\section{The measurement of PE severity}

Only part of the patients received chest computed tomography (CT) scans and the Haller Index were calculated. Besides, all the patients underwent front chestonly optical body surface scan in supine position, in which case the Haller Index could not be obtained and the Depth Ratio (DR) (Fig. 3) were measured as previously described [7] on body surface scan image instead. Every patient underwent initial scan upon ordering of a VB and later follow-ups every 3 months to half a year during use of the device.

\section{Study enrollment and usage of the VB}

Approved by institutional review board and patient consent waived, a retrospective study evaluating outcomes of nonsurgical treatment $\mathrm{PE}$ with the 3D printed VB was performed. Patients were enrolled from March, 2018 to December, 2018. Indications for VB therapy were moderate to severe PE by inspection or CT scan yet too young for surgery, or recurrence after surgery, or aversion to surgery. Contraindications to VB therapy were coagulopathies. All potential candidates for VB treatment were seen and approved or disapproved by a single surgeon. After approval, patients received body surface scan. The collected body surface data were used for VB customization as well as deformity documentation in order to compare with follow-up scans during the course of treatment. By filing online questionnaires one and a half year after the initiation of the study, the patients reported the status of daily VB use (average duration in minutes and times daily), the duration of the treatment (accumulated months used), the vacuum pressures $(\mathrm{kPa})$. The vacuum pressure ranges were recommended according to patients' ages. The VB was first applied 15 min twice daily, and then increased to 30 min twice daily after 2 weeks or more. The whole course of treatment was 1 year and might be extended as appropriate.

\section{Statistical analysis}

Patient demographics, the Haller indices and DRs were collected for statistical analysis. Continuous data following normal distribution were presented in mean \pm standard deviation, or in median and interquartile range if not following normal distribution. Categoric data were present in frequencies and percentages. The initial DRs

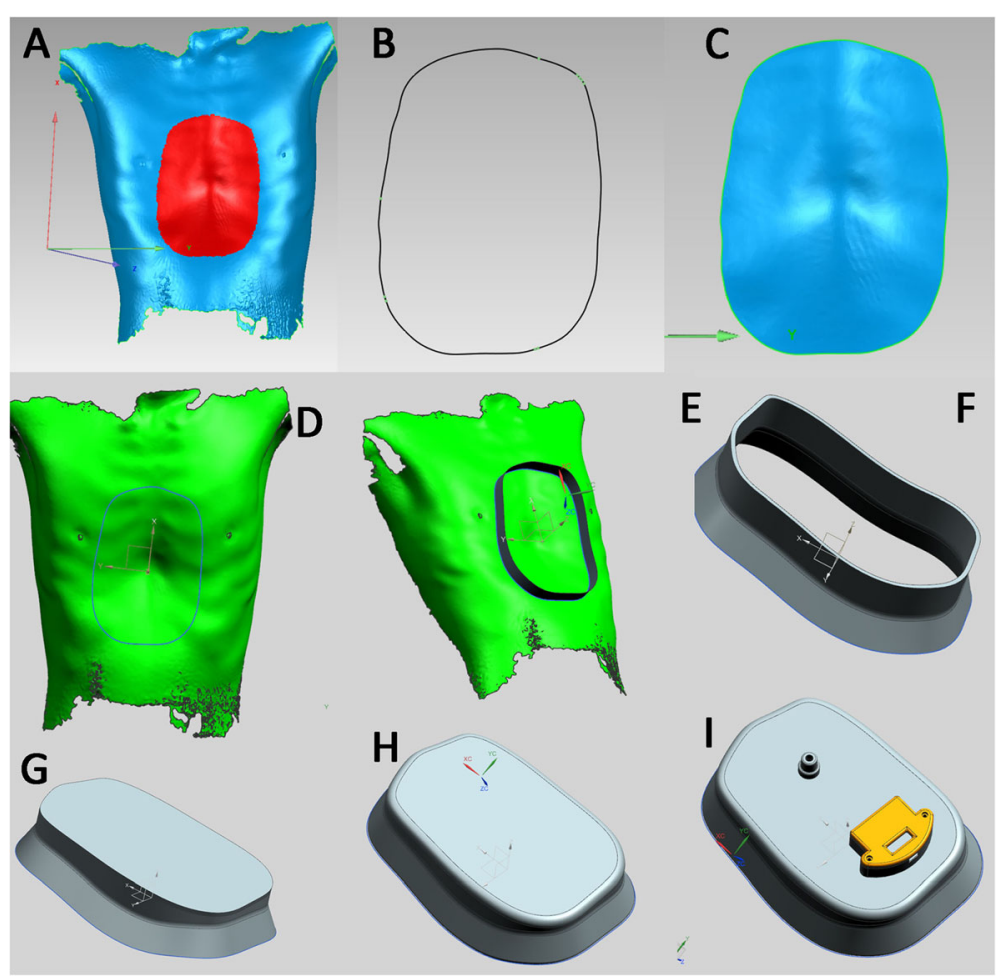

Fig. 1 The steps to design and manufacture a customized silicon part of the 3D printed VB 


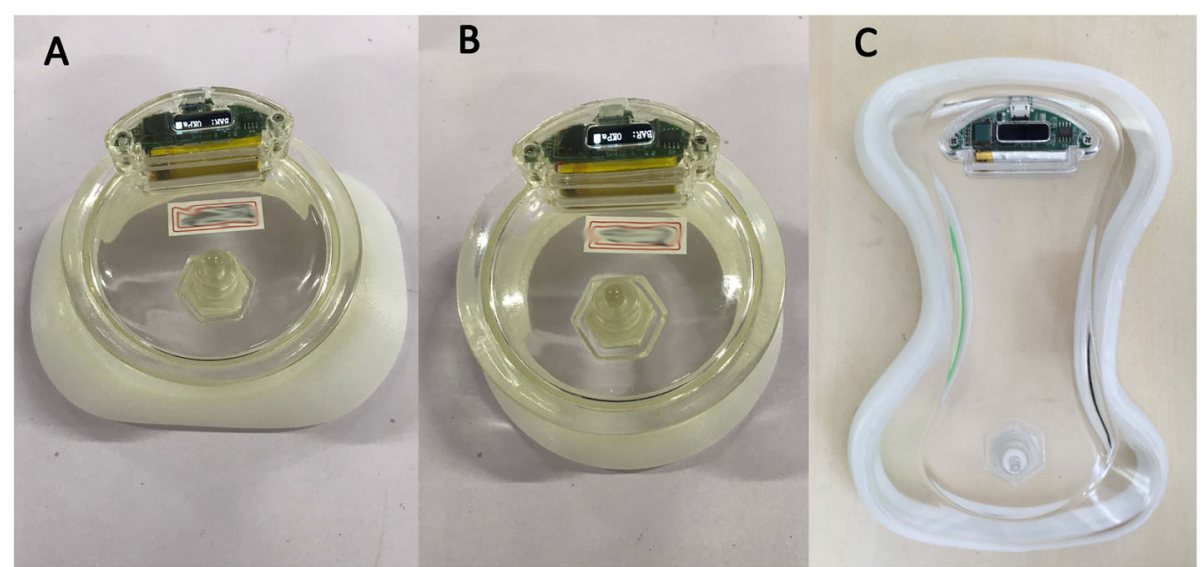

Fig. 2 Different shapes of the 3D printed VBs

and last follow-up DRs were compared using Wilcoxon Signed-rank test or Student $t$ test as appropriate to identify improvement. A broken line plot was also drawn to visualize change in DR over time for patients having had at least one follow-up scan. The correlation between usage duration of the VB and change in DR (last followup DR subtracted by initial DR) over the duration was visualized in scatter plot and analyzed using linear regression. Logistic regression was used to identify factors associated with better effects with VB use. The dependent variable Post-correction Ratio was defined as the ratio of the least DR measured during follow-ups to the initial DR. A cut-off of 0.5 was chosen to transform it into a dichotomous variable. The Post-correction Ratio less than 0.5 were considered more effective of VB use. In univariate analysis $P<0.1$ was chosen as

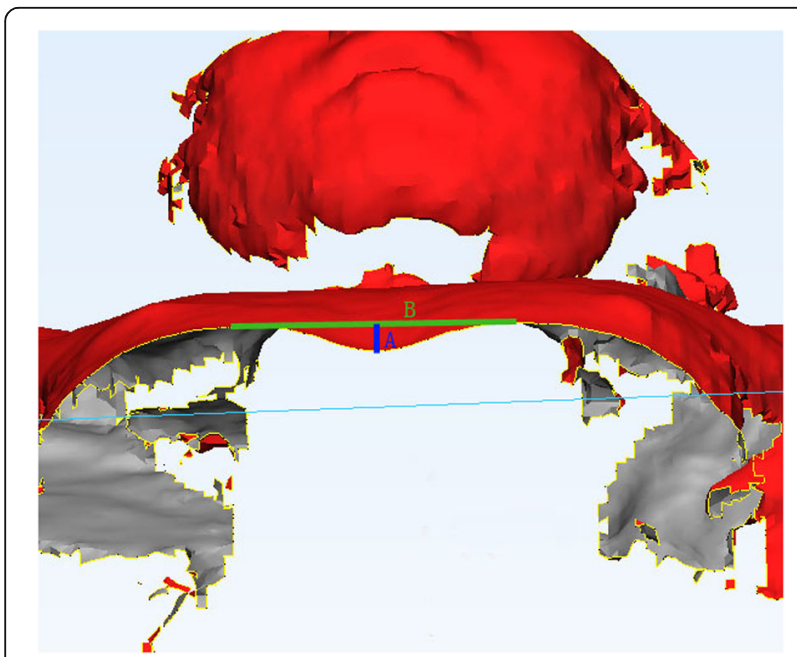

Fig. 3 The measurement of the Depth Ratio, defined as a/b. b: the distance between the highest points on each side of the front chest; a: the shortest vertical distance between external deepest point of the front chest and $b$ inclusion criteria for multivariate step and in multivariate analysis, $P<0.05$ was considered significant. All analyses were conducted using Stata version 15 statistical software (Stata Statistical Software: College Station, TX: Stata Corp LP).

\section{Results}

Forty-two patients (female 16,38.10\%) with a median age of $3.6(2.2,6.2)$ years received nonsurgical treatment with the device. The mean DR at treatment onset was $0.129 \pm 0.036$. Eighteen subjects had undergone a chest CT scan with a mean Haller index of $3.07 \pm 0.42$ (Table 1).

Forty patients/parents completed the questionnaire upon request. The mean follow-up duration was $11.1 \pm$ 3.8 months according to the last body scan date, and change in the DR was $-0.057 \pm 0.023$. With regard to complications, 21(52.5\%) complained petechiae in use, and $12(30 \%)$ reported blistering and some reported pain and/or discomfort. All the symptoms resolved after short time pause. There were no permanent sequelae from side effects. With regards to effectiveness, the majority considered it good $(20,50 \%)$ or fair $(18,45 \%)$. Two patients/parents considered it excellent (Table 2). All patients were still using the VB if not asked to pause or terminate.

Table 1 Demographics of 42 patient receiving vacuum bell therapy

\begin{tabular}{ll}
\hline & Mean \pm SD/median (IQR)/frequency, percentage \\
\hline Age, years & $3.6(2.2,6.2)$ \\
Female & $16,38.10 \%$ \\
Height, $\mathrm{cm}$ & $106.3 \pm 21.4$ \\
Weight, $\mathrm{kg}$ & $21.0 \pm 8.2$ \\
Initial DR & $0.129 \pm 0.036$ \\
Haller index & $3.07 \pm 0.42$ \\
\hline
\end{tabular}


Table 2 Follow-up and questionnaire results

\begin{tabular}{ll}
\hline & Frequency/median (Percent/range) \\
\hline Questionnaire responders & $40(100 \%)$ \\
Dow was the device used? & \\
TIMES per day & $30(15,60)$ \\
Vacuum, kPa & $1.5(1,3)$ \\
Complications & $20(10,30)$ \\
Petechia & $21(52.5 \%)$ \\
Blistering & $12(30 \%)$ \\
Discomfort & $5(12.5 \%)$ \\
Pain & $9(22.5 \%)$ \\
Effectiveness by questionnaire & \\
Poor & $0(0 \%)$ \\
Fair & $18(45 \%)$ \\
Good & $20(50 \%)$ \\
Excellent & $2(5 \%)$ \\
Follow-up & Mean (SD) \\
Duration, months & $11.1(3.8)$ \\
Change in DR & $-0.057(0.023)$ \\
Ratio & $0.54(0.20)$ \\
\hline
\end{tabular}

\# Duration may have changed during treatment. Patients or parents were required to report the parameters they used most

a The ratio of the least DR during usage to initial DR

There were 30 patients having had at least one followup scan and Wilcoxon Signed-rank test shows significant improvement after use of the VBs $(\mathrm{z}=-4.569, p=0.0000)$. All patients achieved better appearance after use though there was some fluctuation as show in the broken line plot (Fig. 4). The scatter plot and fitted line shows correlation between usage duration and change in DR ( $2=0.235$, $p=0.014$ by linear regression, Fig. 5). Significant improvement of the front chest depression of an typical patient using 3D printed vacuum is demonstrated with reconstructed images using body surface scan technique (Fig. 6). After exclusion of 12 patients with no follow-up scan and 5 patients with 10 week or longer pauses, 25 patients with available follow-up scan data were included for logistic regression. In univariate analysis, $\mathrm{p}$ for age and pretreatment DR was $<0.1$ and were included in multivariate analysis. However, none of the two factors was significant in the next step analysis $(p=0.086,0.078$, respectively) (Table 3). At the conclusion of this study, 3 achieved normal front chest appearance and terminated the VB therapy. They were required to visit 3 months later after discontinuation. The rest were still using the device.

\section{Discussion}

Our study has shown the customized 3D printed VB therapy offers nonoperative treatment of $\mathrm{PE}$ at least as effectively as conventional VB though it remained unclear if it results in better outcome at the expense of 3D printing customization. All patients with follow-up data presented better chest appearance confirmed both objectively and subjectively.

Using vacuum suction to lift the sternum was first described more than a century ago, and its usefulness has been confirmed in the treatment of PE over the last 20 years [1-6]. Obviously, one advantage brought by VB therapy compared to surgery is fewer associated risks and less discomfort. However, it remains an alternative rather than a substitute for the Nuss procedure which can achieve excellent results in the majority of patients with one procedure and minimal complications at this stage [5]. A major shortcoming of the VB therapy is that it requires remarkable dedication by the patient and family to keep constant use of the device over at least 1 year or even longer [3] during which many are not able to maintain this compliance. Obermeyer, et al. [8] reported nearly one third of their patients were either lost to follow-up or poorly compliant. Our patients' compliance seemed higher as we required them to visit us every 3 months to half a year since VB onset. And we also kept patients in touch in Wechat, a Chinese social media platform. Regular visits with objective measurement for improvement using body surface scan and inter-parent communication on social media encouraged them to continue the treatment. In the course of treatment as long as at least 1 year, it is very important to have patients back and show them the improvement in the form of reconstructed body surface images and measured numbers. Hence, patients and families may gain confidence in this novel therapy and maintain high compliance.

\section{Effectiveness and side effects}

An excellent correction with VB therapy has been reported from 13.5 to $37.5 \%$ [1, 4, 8, 9] though different definitions of excellence were used. Some use absolute depression depth, which does not account for the body size of patients and requires special tools for measurement, to quantify the correctness, while others use Haller Index, Correction Index or the modified percentage depth [10], all of which require a chest CT scan or special calipers to measure dimensions on body surface. In comparison, as mentioned before, only part of our patients received CT scan and we only body-scanned the front chest of all patients due to insufficient compliance of young patients and also to simplify measurement procedures. Front-chest-only images are enough to customize a VB but makes it impossible to calculate external Haller Index or any other alternative indices. So we measured the DR which accounts for the width of the chest as compared to absolute depression depth. It is 


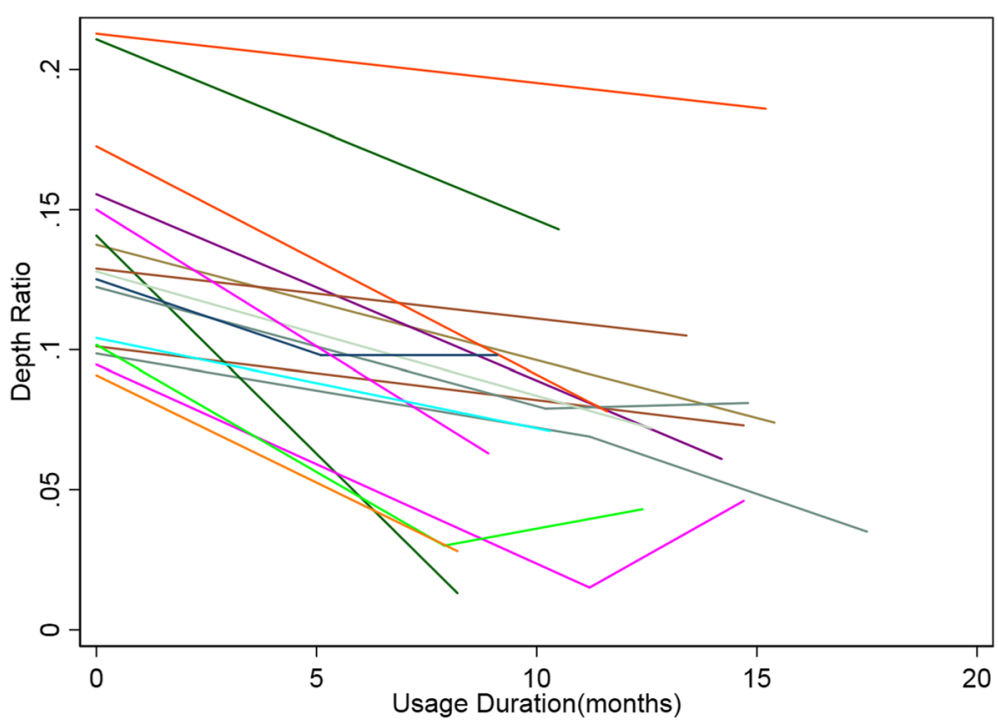

Fig. 4 A broken line plot showing changes in DR over the course of treatment

radiation free, reproducible and needs no additional resource or tools. We have demonstrated its consistency with data measured from chest CT in our previous study [7]. According to the DR, all patients with follow-up data improved in varying degrees. However, we did not use any cut-off of the DR for the definition of excellence outcome. This makes it difficult to compare excellence rates between our series and those in the literature. As per questionnaire data, the majority of the parents considered the effectiveness fair to good. Since most of the patients were still in the course of treatment, the effectiveness was expected to continuously improve. Side effects were as common as reported in the literature, including petechiae, blistering, discomfort or pain $[1,8$, 11]. Although common, all side effects were reversible and resolved by applying reduced suction pressure or pausing for a short period of time, generally no more than 2 weeks. There were no side effects leading to noncompliance.

\section{Outcome related factors}

Previous studies have related several factors with effectiveness of VB use. Obermeyer, et al. [8] found factors predictive of an excellent result being: initial age $\leq 11$ years old, initial chest wall depth $\leq 1.5 \mathrm{~cm}$, good chest wall flexibility, and usage duration more than 12 months. They noticed that several patients used below $60 \mathrm{~min}$ per day and still achieved an excellent correction, which was in contrast to Lopez et al., who identified optimal use of $4 \mathrm{~h}$ or more per day [4]. However, both of them argued that chest wall flexibility is a significant indicator of success. Though we did not find any factor associated

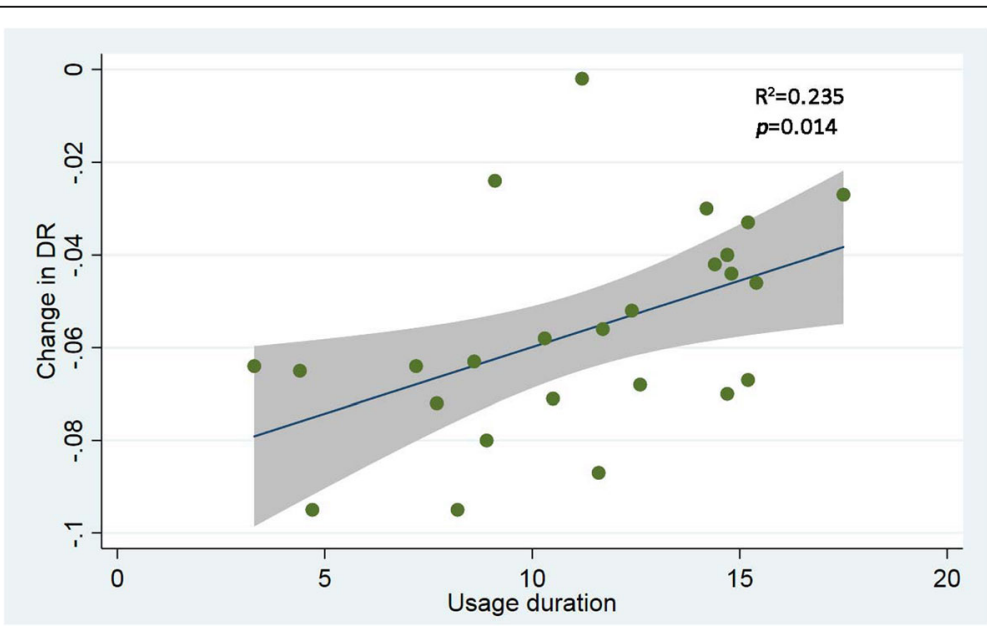

Fig. 5 The scatter plot and fitted line shows a correlation between usage duration and change in $D R, R^{2}=0.235, p=0.014$ by linear regression 


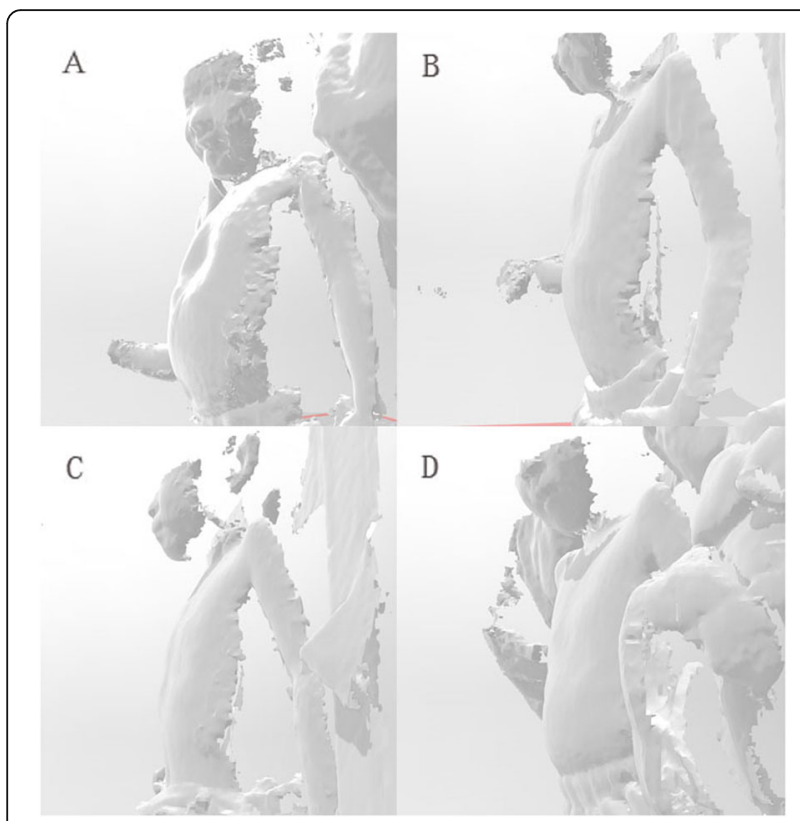

Fig. 6 An example of typical patients using 3D printed vacuum and having demonstrated significant improvement of the depression of the front chest. $\mathbf{a}$ : Started to use the 3D printed VB; $\mathbf{b}$ : 3 months after use; $\mathbf{c}$ : 9 months after use and stopped VB therapy; $\mathbf{d}$ : 3 months after termination of VB use

with better correction of the PE, there was a trend for patients with younger age and smaller DR (less severity) to have better outcomes according to our data. And patients in our study much younger than those in the two publications mentioned above may explain why we had achieved remarkable improvement with only $30 \mathrm{~min}$ per time and 1.5 times daily use, which was much shorter than anyone reported before. We did not included flexibility as an analytical factor as first, it was difficult to quantify it and second, we usually consider the chest of a population with a median age of 3.6 years flexible, which was evidenced by a median suction vacuum of only $20 \mathrm{kPa}$. Obviously, chest flexibility is closely associated with age. It could not be ruled out that these two may interact as confounders when performing regression analysis, especially when all previous studies are of small sample sizes.

\section{Timing of treatment}

With regard to timing for treatment, it once was indicated for preschoolers to have Nuss procedure and then switched to teenagers/adolescents as an optimal age for surgery in view of higher recurrence if patients were operated on very young [12]. For VB therapy, Obermeyer, et al. [8] concluded VB therapy should be considered at approximately 11 years of age. Since if the correction is inadequate after 12 to 18 months of VB therapy, surgery could be performed in the optimal timing range for the Nuss procedure. We believe, however, a conservative therapy like VB can be tried as young as toddlers or preschoolers like our cases for current data support better outcomes in younger patients with more flexible chest, especially in patients with deep depression in whom treatment could not be refrained until later. Though we do not yet know the recurrence rate of our series due to short follow-up, it might be expected to have higher recurrence with experience from surgery at very young ages. However, a VB therapy is of minimum major complications, and could be repeated if necessary, without worry about osteochondrodystrophy or other major complications of surgery. It may be worth it to try at a young age to avoid years of waiting during which heart and respiratory impair may occur.

\section{Advantage of $3 \mathrm{D}$ and the cost}

The prominent difference of the device we used in this study from conventional VBs, as we mentioned before, is the 3D printing customization. Basically, our device

Table 3 Logistic regression for better corrective effects of PE

\begin{tabular}{|c|c|c|c|c|c|c|}
\hline \multirow[t]{2}{*}{ Variable } & \multicolumn{3}{|l|}{ Univariate } & \multicolumn{3}{|c|}{ multivariate } \\
\hline & $\overline{\mathrm{OR}}$ & $95 \% \mathrm{Cl}$ & $p$ value & $\overline{\mathrm{OR}}$ & $95 \% \mathrm{Cl}$ & $P$ value \\
\hline$\overline{\text { Age }}$ & 1.37 & $(0.96,1.95)$ & 0.084 & 1.40 & $(0.95,2.07)$ & 0.086 \\
\hline Sex & 1.60 & $(0.33,7.85)$ & 0.562 & & & \\
\hline Height & 1.03 & $(0.99,1.08)$ & 0.115 & & & \\
\hline Weight & 1.07 & $(0.95,1.20)$ & 0.268 & & & \\
\hline Initial DR & $3.43 e+11$ & $(0.02,7.69 e+24)$ & 0.090 & $3.40 e+11$ & $(0.05,2.37 \mathrm{e}+24)$ & 0.078 \\
\hline Duration between treatment onset and last follow-up & 1.20 & $(0.95,1.51)$ & 0.130 & & & \\
\hline Total hours of VB usage & 1.00 & $(1.00,1.01)$ & 0.425 & & & \\
\hline Vacuum pressure & 1.02 & $(0.85,1.2)$ & 0.854 & & & \\
\hline Times per day & 0.60 & $(0.14,2.55)$ & 0.491 & & & \\
\hline Hours of VB usage daily & 1.05 & $(0.96,1.14)$, & 0.263 & & & \\
\hline Total days VB used & 1.00 & $(0.99,1.01)$ & 0.894 & & & \\
\hline
\end{tabular}


draws on the same physical principle as other VBs reported in the literature. Theoretically, a 3D contact surface configuration may warrant better airtightness in application, which is especially true in patients with breasts developing or asymmetrical pathology. We did not use a conventional VB for comparison in our study, and the configuration of the depression may have changed over the course of treatment, leaving the initial airtightness in question. Therefore, the superiority in airtightness of this devices needs to be confirmed in further studies. The cost of a customized VB is 6000 RMBs, approximately double the price of a conventional VB in China, but still much cheaper than a Nuss procedure, which would cost 25,000 RMBs. We think it is worth trying in view of published results of conventional VBs and the cost.

\section{Limitation}

This study is limited in that it is a retrospective case series without control. And there were some patients who were lost to follow-up, poorly compliant, or had insufficient data. There were also some other limitations. First, questionnaire data with regard to daily use and pressure were self-reported and could not rule out memory-related or other errors. Second, the pressure applied varied according to age and stage of use, so relevant data were represented by values used most for each patient. Third, since our patients were generally young and the majority had passed through a winter during the course of treatment, quite a few paused for several weeks or even months. Though parents were required to report these pauses, errors might have occurred when calculating accumulated duration. Finally, as only part of the patients underwent chest CT and had the Haller index data available, we did not use the Haller index or depth for analysis. Instead, we used the DR as a surrogate, which has never been used in any of previous studies, making it impossible to conduct direct comparison with data in the literature.

\section{Conclusion}

Our initial experience has shown the $3 \mathrm{D}$ printed VB may be as effective as other conventional VBs and could be used as an alternative to surgical treatment for selected patients with PE. More experience and studies with this type of VB are needed to demonstrate its superiority with regard to the $3 \mathrm{D}$ printing design and optimal timing and indication for use.

\section{Abbreviations}

PE: Pectus excavatum; VB: Vacuum bell; CT: Computed tomography; DR: Depth Ratio; 3D: Three dimensional

\section{Authors' contributions}

$X D, P H, J L, J W, L Y$, and $G Y$ contributed to the data collection of the manuscript. XD, SH contributed to the study idea and conceptualization of the manuscript. $X D, P H, J L, J W, L Y$, and GY performed the surgeries. $X L, S X$ provide support for statistical analysis. All authors contributed to revisions of the manuscript. All authors read and approved the final manuscript.

\section{Funding}

This project was supported by the Natural Science Foundation of Hunan Province, China (Grant No. 2019JJ80106).

\section{Availability of data and materials}

The datasets used are available from the corresponding author on reasonable request.

Ethics approval and consent to participate

This study was approved by the Ethics Committee of Hunan Children's Hospital.

Consent for publication

Waived by Ethics Committee of Hunan Children's Hospital.

\section{Competing interests}

The authors declare that they have no competing interests.

\section{Author details}

'Deaprtment of Cardiothoracic Surgery, Hunan Children's Hospital, No. 86 Ziyuan Road, Changsha 410007, Hunan, China. ${ }^{2}$ Deaprtment of Radiology, Hunan Children's Hospital, Changsha 410007, Hunan, China. ${ }^{3}$ Pediatrics Research Institute of Hunan Province, Hunan Children's Hospital, 86 Ziyuan Road, Changsha, China.

Received: 8 March 2020 Accepted: 1 September 2020

Published online: 10 September 2020

\section{References}

1. Haecker FM. The vacuum bell for conservative treatment of pectus excavatum: the Basle experience. Pediatr Surg Int. 2011;27(6):623-7.

2. Haecker FM, Mayr J. The vacuum bell for treatment of pectus excavatum: an alternative to surgical correction? Eur J Cardiothorac Surg. 2006;29(4):557-61.

3. Haecker FM, Sesia S. Vacuum bell therapy. Ann Cardiothorac Surg. 2016;5(5): 440-9.

4. Lopez M, Patoir A, Costes F, Varlet F, Barthelemy JC, Tiffet O. Preliminary study of efficacy of cup suction in the correction of typical pectus excavatum. J Pediatr Surg. 2016;51(1):183-7.

5. Nuss D, Obermeyer RJ, Kelly RE. Nuss bar procedure: past, present and future. Ann Cardiothorac Surg. 2016;5(5):422-33.

6. Togoro SY, Tedde ML, Eisinger RS, Okumura EM, de Campos JRM, PegoFernandes PM. The vacuum bell device as a sternal lifter: an immediate effect even with a short time use. J Pediatr Surg. 2018:53(3):406-10.

7. Deng X, Huang P, Luo J, Wang J, Yi L, Yang G, et al. The consistency of an optical body surface scanning method compared with computed tomography: a validation study. J Pediatr Surg. 2020;55(8):1448-52.

8. Obermeyer RJ, Cohen NS, Kelly RE Jr, Ann Kuhn M, Frantz FW, McGuire MM, et al. Nonoperative management of pectus excavatum with vacuum bell therapy: a single center study. J Pediatr Surg. 2018;53(6):1221-5.

9. Schier F, Bahr M, Klobe E. The vacuum chest wall lifter: an innovative, nonsurgical addition to the management of pectus excavatum. J Pediatr Surg. 2005:40(3):496-500

10. Snyder CW, Farach SM, Litz CN, Danielson PD, Chandler NM. The modified percent depth: another step toward quantifying severity of pectus excavatum without cross-sectional imaging. J Pediatr Surg. 2017;52(7):1098-101.

11. St-Louis E, Miao J, Emil S, Baird R, Bettolli M, Montpetit K, et al. Vacuum bell treatment of pectus excavatum: an early north American experience. J Pediatr Surg. 2019:54(1):194-9.

12. Nuss D, Obermeyer RJ, Kelly RE Jr. Pectus excavatum from a pediatric surgeon's perspective. Ann Cardiothorac Surg. 2016;5(5):493-500.

\section{Publisher's Note}

Springer Nature remains neutral with regard to jurisdictional claims in published maps and institutional affiliations. 